\title{
A giant X-ray flare from a BO + Post-T Tauri system detected by EXOSAT
}

\author{
G. Tagliaferri ${ }^{1}$, P. Gionmi ${ }^{1,3}$, L. Angelini ${ }^{1}$, J.P. Osborne ${ }^{1}$ \\ and \\ R. Pallavicini ${ }^{2}$
}
${ }^{1}$ EXOSAT Observatory, Astrophysics Division, Space Science Department of ESA, ESTEC, The Netherlands.
${ }^{2}$ Osservatorio Astrofisico di Arcetri, Firenze, Italy.
${ }^{3}$ On leave of absence from: CNR, Istituto di Fisica Cosmica, Milano, Italy.

\begin{abstract}
We report the serendipitous discovery by EXOSAT of a flaring $\mathrm{X}$-ray source in the field of the Seyfert type I galaxy III ZW 2. We identify this source with the visual binary HD 560 (B9V + G5Ve) and argue that virtually all of the observed X-ray flux, including the flare, came from its late-type component (HD $560 \mathrm{~B}$ ). Optical studies have lead to the identification of HD $560 \mathrm{~B}$ as a post-T Tauri star. Since these stars are difficult to detect by optical methods, X-ray observations may prove to be the best way to identify them.
\end{abstract}

\section{Introduction}

The Low Energy (LE) Imaging Telescopes on the EXOSAT satcllite (ref. 21) have proved to be very good instruments for detecting serendipitous sources in the soft X-ray energy band $0.05-2.0 \mathrm{keV}$ (ref. 5). In particular, the relatively long ( $\geq 10^{4}$ seconds) and uninterrupted EXOSAT observations allow the variability of these new $\mathrm{X}$-ray sources to be studied on time scales ranging from seconds to several hours. Here we present the serendipitous detection of X-ray emission from the visual binary IID 560, consisting of a B9V $\left(m_{v}=5.53\right)$ stin and G5Ve $\left(m_{v}=10.37\right)$ star, with a separation of 7.7 aresec (ref. 4). This systcm was seen in the field of the Seyfert type I galaxy III ZW 2, observed four times by EXOSAT. During one of the observations (1985 November 30 ), the serendipitous source was observed to flare in both tlie LE and ME experiments (fig. 1; ref 20).

\section{Data analysis}

In the LE the flare started at $~ 03: 48$ UT with the flux increasing more than a factor of thrce in $\sim 40 \mathrm{~min}$. The decay is exponential-like, but $\sim 50 \mathrm{~min}$ after the peak a liump is seen in the light curve (fig. 1). The source was also included at $\sim 85 \%$ efficiency in the collimator response of the ME experiment, a large area proportional counter sensitive in the 1-15 keV energy band, with a field of view of 45 arcmin FWIM (ref. 22). The ME light curve on 1085 Nov 30 is also shown in fig. 1. It is generally dominated by the flux from III 
ZW 2, and is constant except for a period starting at 03:55 UT and ending at 05:35 UT when a strong flare is seen, similar in shape to the one detected simultaneously in the LE light curve of the serendipitous source. The rise of the flare in the ME is somewhat faster than in the soft energy band and the flare peaks a few minutes earlier. A hump during the decay phase is seen, which occurs some minutes before that in the LE (fig. 1). A cross correlation analysis shows that the delay between the flux in the two experiments (LE delayed with respect to ME) was $6.0 \pm 1.1$ minutes $(90 \%$ confidence). We note that an earlier peak in hard $X$-rays than in soft $\mathrm{X}$-rilys is usually observed in solar and stellar flares and can be easily understood as due to rapid heating and subsequent cooling of the hot flare plasma. HD 560 was also serendipitously detected on two occasions by the IPC experiment on EINSTEIN sitellite. The observed IPC count rates were $5.5 \times 10^{-2}$ and $4.4 \times 10^{-2}$ cts $s^{-1}$ respectively (ref. 9), which correspond to a luminosity of $\sim 8 \times 10^{29} \mathrm{erg} \mathrm{s}^{-1}$, obtained using a conversion factor of $2 \times 10^{-11} \mathrm{erg} \mathrm{cm} \mathrm{count}^{-1}$ (ref. 23) and a distance for this system of 81 parsec (ref. 8 ).

The EXOSAT quiescent X-ray luminosity in the band $0.05-2.0 \mathrm{keV}$, assuming an optically thin line + continuum thermal model (ref. 10), wilh typical coronal temperatures ranging from 0.3 to $1.0 \mathrm{keV}$, is between 2.2 and $2.5 \times 10^{30} \mathrm{crg} \mathrm{s}-1$. The ME flare spectrum has been integrated from 03:50 UT to 05:30 UT and titted with a thermal model (ref. 10). The best fit temperature is $3.9 t_{+1.5}^{-0.9} \mathrm{keV}$ (errors have $90 \%$ confidence, $x_{\min }^{2}=20$ for 23 (d.o.f.),

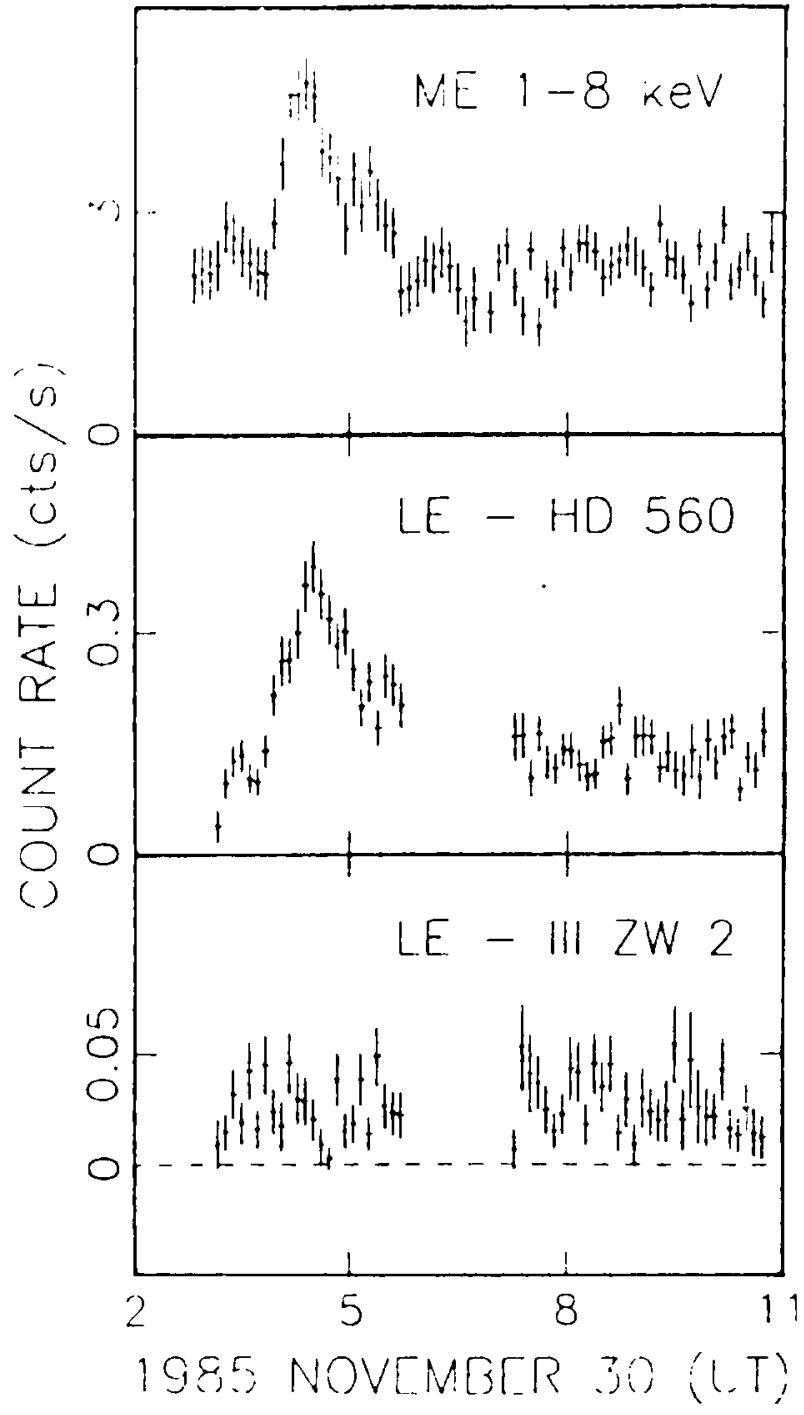

FIG.1 EXOSAT observation of the field of III ZWV 2 on November 301985 . From top to bottom: a) ME light curve for the field containing both the Seyfert galaxy and the serendipitous source; b) LE light curve of the serendipitous source; c) LE light curve of III Z $\mathrm{IV}^{\prime}$ 2. All light curres have been background subtracted. the corresponding emission measure is $1.5 \times 10^{54} \mathrm{~cm}^{-3}$. For the fit we fixed the interstellar column density $\left(N_{H}\right)$ at $1 . S \times 10^{19} \mathrm{~cm}^{-2}$, the value expected for a wiform interstellar density of $0.07 \mathrm{~cm}^{-3}$ (ref. 
15). Leaving $N_{H}$ as a free parameter in the fit procedure we obtained a temperature of $4.1 \mathrm{keV}$ and $N_{H}$ of $3.9 \times 10^{18} \mathrm{~cm}^{-2}$. The total flare energy between 0.05 and $10 \mathrm{keV}$ is $1.9 \times 10^{35}$ ergs.

\section{Discussion}

We have reported the discovery by EXOSAT of a flaring X-ray source in the field of the Seyfert galaxy III ZW 2 and we have identified the flaring source with the visual binary HD 560 which contains a B9 V primary and a G5 Ve secondary. The EXOSAT and EINSTEIN observations do not have the angular resolution to discriminate between the two stars. However the extensive stellar surveys carried out with the EINSTEIN Observatory (ref. $16,19)$ have shown that B8-A5 stars as a class are not X-ray emitters at levels greater than $\sim 10^{27} \mathrm{erg} \mathrm{s}^{-1}$. Recently $\mathrm{X}$-ray emission has been detected from a few late- $\mathrm{B}$ stars in regions of star formation (ref. 1,26). Whether or not the $\mathrm{X}$-ray emission in these cases can be properly attributed to the hot stars or to hidden late-type companions remains to be determined. However in our case we know that a young late type companion star is present. The special interest of this observation is that the late-type star HD $560 \mathrm{~B}$ has been suggested to be a post-T Tauri star (PTTS) (ref. 8), i.e. a star still contracting toward the main-sequence and intermediate between classical $\mathrm{T}$ Tauri stars and ZAMS stars. Pre-main-sequence (PMS) stars are known to show flares (ref. 2, 3, 11), and to have $\mathrm{X}$-ray luminosities in the range $10^{30}-10^{31} \mathrm{erg} \mathrm{s}^{-1}$ (ref. 12, 25). We are not aware of any report of an X-ray flare from an early type (O-B) star. Are the observed X-ray properties consistent with the identification of HD $560 \mathrm{~B}$ as a PTTS?

The quiescent X-ray luminosity derived for HD $560 \mathrm{~B}$ is comparable to that of the PTTS candidates observed by EINSTEIN (ref. 12, 25). The X-ray luminosity of all these sources is $\sim 10^{30} \mathrm{erg} \mathrm{s}^{-1}$, i.e. much higher (by a factor 10 to 1000) than typical Xray luminosities of late-type main-sequence stars and subgiants (ref. 16), except for the special case of close binaries of the RS CVn type. Another source having comparable X-ray luminosity is AB Dor (HD 36705) (ref. 2,13) for which some evidence has recently been produced (although not conclusive as yet) that it might be a pre-main sequence object, possibly a PTTS (ref. 17, 18,25). The total energy released in X-rays by the flare on HD $560 \mathrm{~B}$ was $\sim 10^{35} \mathrm{erg}$, which is larger (by at least a factor of 10) than the highest total energies typically released by flares of main-sequence stars (ref. 14). This energy again is comparable to the total X-ray energy released by large flares on RS CVn and Algol-type binaries (ref. 27) and is indicative of an extreme level of activity. The high quiescent X-ray luminosity, the flaring belaviour and the large energy released in the flare, all indicate that this is a star with a very active corona and has X-ray characteristics typical of PMS stars. This is consistent with the results of optical studies which have identified it as a member of the elusive class of post-T Tauri stars. These were first proposed as a class of PMS objects by Herbig $(1978$, ref. 6), who noticed that the T Tauri phase occupies only a small fraction (about 5 to $10 \%$ ) of the contraction time of a $1 \mathrm{M}_{\odot}$ star toward the main sequence. As a consequence, many more PTTS should exist than classical T Tauri stars. Unfortunately it is quite difficult to detect PTTS by optical methods and X-ray observations may prove to be the best way to identify them. To conclude, the $\mathrm{X}$-ray emission from HD 560 is 
most likely from the G5Ve star not its B9 companion, bringing further support to the identification of $\mathrm{HD} 560 \mathrm{~B}$ as a PMS star and sheding light on the X-ray properties of PTTS.

\section{References}

1) Caillault, J.-P. and Zoonematkermani, S.: 1987, Circumstellar Matter (I. Appenzeller and C. Jordan eds.), p.110.

2) Collier Cameron, A., ct al.: 1088, M.N.R.A.S 231, 131.

3) Feigelson, E.D., and DeCampli, W.M.: 1981, Ap. J. (Letters) 243 L89.

4) Giahm, G.F., et al.: 1983, Astron. Ap. Suppl., 51, 143.

5) Giommi, P., et al.: 1088, in "X-Ray Astronomy with EXOSAT", (N.E. White and R. Pallavicini eds.), Memorie S.A.It., 59, n.1, in press.

6) Herbig, G.H.: 1978, in "Problems of Physics and the Evolution of the Universe" (L.V. Mirzoyan ed.), p. 17.1.

7) Lindroos, K.P., 1985, Astron. Ap. Suppl., 60, 183.

8) Lindroos, K.P., 1980, Astr. Ap., 156, 223.

9) Maccacaro, T., et al.\& 1987, Astron. J., 93, 1484.

10) Mewe, R., ct al.: 1985, Astron. Ap. Suppl. , 62, 197.

11) Montmerle, T., et al.: 1983, Ap. J., 269, 182.

12) Mundt, R., et al.: 1083, Ap. J., 269, 229.

13) Pakull, M.: 1981, Astron. Ap., 104, 33.

14) Pallavicini, R.: 1988, in "Solar and Stellar Coronal Structure and Dynamics" (R. Altrock ed.), in press.

15) Paresce, F.: 1084, Astron. J., 89, 1022.

16) Rosner, R., et al.: 1985, Ann. Rev. Astr. Ap., 23, 413.

17) Rucinski, S.M.: 1982, Inf. Bull. Var. Stars, No. 2203.

18) Rucinski, S.M.: 1985, M.N.R.A.S., 215, 591.

19) Schmit,, J.H.M.M.: 1088, Iot Thin Plasmas in Astrophysics (R. Pallavicini ed.), in press.

20) Tagliaferri, G., et al.: 1988, Ap. J. (Letters), 331, L113.

21) Taylor, B.G., et al.: 1081, Space Sci. Rev., 30, 479.

22) Turner, M.J. L., et al.: 1081, Space Sci. Rev., 30, 513.

23) Vaiana, G.S., et al. 1981, Ap. J., 245, 163.

24) Vilhu, O., et a!.: 1987, Ap. J., 320, 850.

25) Walter, F.M.: 1986, Ap. J., 306, 573.

20) Walter, F.M., et al.: 1988, A. J., 96, 297

27) White, N.E., et al.: 1986, Ap. J., 301, 262. 